\title{
THE USEFULNESS OF MOBILE COMPUTED TOMOGRAPHY IN A RURAL AREA
}

\author{
Mitsuhiro Kamiyoshihara, Susumu Ishikawa, Yasuo Morishita \\ Second Department of Surgery, Gunma University School of Medicine
}

\begin{abstract}
Introduction : Nakazato village is located in the far-southwest region of Gunma prefecture. It takes at least one hour to go to the nearest general hospital. Author's clinic does not have a CT scanning device. Until that time, every patient went to far hospitals by oneself, when CT scan was necessary for further examination. This study adverted to the usefulness of Mobile CT in a rural clinic. Patients and Methods : Between June 1995 and October 1997, 20 patients needed CT scanning because of further examination. The mean age was 72 years old, and the ratio of male to female was 9 to 11 . Results : Seven patients had surgical treatment for cancer, and we had suspected recurrence of primary cancer. Four patients needed CT scanning in following various diseases. Nine patients had an abnormal shadow on chest roentgenogram. The disease was detected in 8 patients $(40 \%)$ out of 20 patients. Of 7 postoperative cancer patients, 2 had primary cancer recurrence, each of which was lung cancer and breast cancer. Of the remaining 9, 6 patients had a disease, which was chronic bronchitis in 2 patients, and bronchoectasia, emphysema, lung abscess and pleuritis in 1 each. Conclusion : Using the mobile CT makes it easy for patients to take further examination. We deliberate that this device is useful and recommends for rural clinics or hospitals, which do not have a CT scanning device.
\end{abstract}

Key words : Mobile computed tomography, Rural area, Rural clinic, Follow-up, Out-patient (Kitakanto Med.J. 48 (4) : 287 288, 1998)

\section{INTRODUCTION}

Nakazato village is located in the far-southwest region of Gunma prefecture. It takes at least one hour to go to the nearest general hospital. Author's clinic is only a medical institute in this village. This clinic has a bronchoscope, a gastroscope, a colonoscope, a roentgenogram device, an ultrasonogram device and an electorocardiogram device, but does not have a CT scanning device. Until that time, every patient went to far hospitals by oneself, when CT scan was necessary for further examination. As we dissolved the inconvenience, mobile $\mathrm{CT}$ was introduced. This study adverts to the usefulness of mobile $\mathrm{CT}$ in a rural clinic.

\section{PATIENTS AND METHODS}

Between June 1995 and October 1997, 20 patients were contemplated that $\mathrm{CT}$ scanning was necessary because of further examination. The mean age was 72 years old, and the ratio of male to female was 9 to 11 . All studies were performed on the mobile CT car, which had X-vision TSX-002 $\mathrm{A}^{\mathrm{TM}}$ (Toshiba Medical, Tokyo) as CT scanning device. Scanned sections were $1 \mathrm{~cm}$ thick. Scanning of the chest was performed in suspended inspiration. None of the patients received iodinated contrast medium intravenously for safety.

\section{RESULTS}

Of these, 7 patients after surgical treatment for cancer (lung cancer in 3 patients, breast cancer in 2 and colon cancer in 2), and we had suspected recurrence. Four patients needed CT scanning in following various diseases: pancreatic cyst in 2 patients, hepatoma in 1, abdominal aortic aneurysm in 1. Nine patients had an abnormal shadow on chest roentgenogram.

The disease was detected in 8 patients $(40 \%)$ out of 20. Of 7 postoperative cancer patients, 2 had primary cancer recurrence, each of which was lung cancer and breast cancer. Of the remaining 9 further examination patients, 6 had a disease, which was chronic bronchitis in 2 , bronchoectasia in 1 , emphysema in 1 , lung abscess in 1 , and pleuritis in 1 . 


\section{DISCUSSION}

Nakazato village is located in the far-southwest region of Gunma prefecture. It takes at least one hour to go to the nearest general hospital. This village has a small population : 1150 residents. Year after year, this village gets depopulated, and the mean age of the residents makes a change for the older. Two-hundred sixty-one residents $(40.8 \%)$ of these are the advanced age people of 70 years old or over, almost all people of whom cannot drive a car. Only means of transportation is a bus, and there is no railroad. Therefore, every patient who needed a CT did not always take further examinations.

Cancer is the major cause of death in patients aged 70 years and over. The life expectancy in Japan, as of 1995 , was advanced from age 70 years to about 82.47 years for men and about 86.75 years for women; the corresponding increases from the age 80 years are 7.14 and 9.47 years, respectively ${ }^{1)}$. Therefore, the number of patients 70 years of age or older with cancer is contemplated to increase.

In the future, the number of elder patients in rural area will be expected to increase. It is necessary not always to detect the disease in the elders as early as possible, but to follow up them after surgical treatment. These are one of the important role for rural clinics $^{2}$. Under this environment, the follow-up of the postoperative patients has bothered us. In usual means, we have examined them with the tumor markers, the ultrasonogram device and the physical findings. Additionally, we recommended the patients who were suspected of having cancer recurrence to take a CT scanning at general hospital ${ }^{3}$. However, more
Fig. 1 Clinical course

\begin{tabular}{|c|c|c|}
\hline \multicolumn{3}{|l|}{ Recurrece of primary } \\
\hline Lung cancer & $3-$ & Metastasis of \\
\hline Breast cancer & 21 & primary cancer \\
\hline Colon cancer & 2 & \\
\hline Follow-up of disease & 5 & \\
\hline Pancreatic cyst & 2 & Chronin bronchitis \\
\hline Hepatoma & 1 & Bronchoectasia \\
\hline Abdominal aorticaneurysm & 1 & Emphysema \\
\hline Abnormal shadow on chest & & Lung abscess \\
\hline $\mathrm{X}-\mathrm{P}$ in a medical check & & -Pleuritis \\
\hline
\end{tabular}

than $40 \%$ of the elders lived by oneself or were nuclear family who did not have meant of a transportation. Therefore, it was uneasy for the elderly patients to go to general hospitals.

Using mobile CT makes it easy for patients to take a further examination. Additionally, several diseases were detected in the 8 patients $(40 \%)$ out of 20 . We deliberate that this device was useful and recommended for rural clinics or hospitals.

\section{REFERENCES}

1) Ministry of Health and Welfare of Japan. Vital statistics (in Japanese). Tokyo: Health and Welfare and Welfare Statistics Association, 1996 : 429.

2) Mobile technology in rural hospitals: the case of the CT scanner. Hartley D, Moscovice I, Christianson J. Health Serv Res, 1996 ; 31 : 213-34.

3) Technical note: mobile CT scanner gantry for use in the operating room. Okudera $\mathrm{H}$, Kobayashi $\mathrm{S}$, Sugita K. Am J Neuroradiol, 1991; 12 : 131-2. 